\title{
Full characterization of a phase-locked DUV double pulse generated in an all-reflective shaping setup working under grazing incidence in a broad spectral range
}

\author{
S. Hartwell ${ }^{1}$ A. Azima ${ }^{2}$. C. Haunhorst ${ }^{3} \cdot$ M. Kazemi ${ }^{1} \cdot$ M. Namboodiri $^{1} \cdot$ A. Przystawik ${ }^{1} \cdot$ D. Schwickert ${ }^{1}$. \\ S. Skruszewicz ${ }^{1}$ D. Kip ${ }^{3} \cdot$ M. Drescher ${ }^{2} \cdot$ T. Laarmann ${ }^{1,4}$
}

Received: 20 April 2021 / Accepted: 12 November 2021 / Published online: 7 December 2021

(c) The Author(s) 2021

\begin{abstract}
Controlling the temporal and spectral properties of ultrashort laser pulses in the visible and near-infrared spectral range by means of a femtosecond pulse-shaping device is a powerful tool with many applications in ultrafast spectroscopy. A major and successful concept is known as the $4 f$ design, which has a symmetric zero-dispersion-compressor geometry. Most $4 f$ pulse shapers rely on using transmissive optics in their beam path limiting the operational wavelength ranges. In the present contribution, we use an all-reflective shaping setup to generate a phase-locked $266 \mathrm{~nm}$ double pulse to benchmark its performance in the limit of short wavelengths. The setup comprises the complete spectral amplitude and phase diagnostics for quantitative analysis of the pulse properties before and after the shaper using the technique of frequency-resolved optical gating. The measured time-frequency spectra are in good agreement with optical simulations. The geometry and hardware of the device including the optical components are designed, such that all harmonics of the deep UV pulses travel the same path, giving the instrument the ability to work with soft X-ray pulses, under vacuum conditions, down to the few-nanometer wavelength scale.
\end{abstract}

\section{Introduction}

Conventional $4 f$ pulse shapers typically utilise several transmissive optical components in their beam path for focusing and pulse manipulation. The latter can be realized, for instance, by an acousto-optical modulator (AOM), where a piezoelectric transducer creates sound waves in a material like germanium, or by spatial light modulators (SLM) based on pixelated liquid-crystal technology allowing for

S. Hartwell

samuel.hartwell@desy.de

T. Laarmann

tim.laarmann@desy.de

1 Deutsches Elektronen-Synchrotron DESY, 22607 Hamburg, Germany

2 Department of Physics, University of Hamburg, 22761 Hamburg, Germany

3 Faculty of Electrical Engineering, Helmut Schmidt University, 22043 Hamburg, Germany

4 The Hamburg Centre for Ultrafast Imaging CUI, 22761 Hamburg, Germany spectral amplitude and phase control [1-3]. Such pulseshaping devices are standard spectroscopic tools in many femtosecond laser laboratories worldwide and commercially available. They work well within their designated frequency domains offering full control over the laser pulse properties [4]. However, the use of transmissive optical components restricts corresponding laser applications to wavelengths longer than $200 \mathrm{~nm}$ [5], as typical optics materials absorb strongly below. Striving to push the short-wavelength frontier of pulse-shaping technology further down, the use of reflective optics is an alternative. Special pixelated micromirror arrays, the so-called micro-electro-mechanical systems (MEMS), have been applied successfully to shape femtosecond pulses at a central wavelength of $200 \mathrm{~nm}[6,7]$. Meanwhile, it has been demonstrated that these advanced reflective optics also perform well in the 30-90 nm spectral range [8]. Note that the application of MEMS technology beyond the extreme ultraviolet spectral range is mainly limited by the slope error and overall surface roughness between individual pixels, which are on the $10-\mathrm{nm}$ scale. The phase errors caused by these imperfections have to be a fraction of the used wavelength to allow for interferometric precision in sculpting waveforms at will [9]. 
Here, we discuss the performance of an all-reflective $4 f$ pulse shaper operating at short wavelength in grazing incidence using a femtosecond $266 \mathrm{~nm}$-deep ultraviolet (DUV) pulse to test the functionality of the device and displaying the feasibility of such a system. The pulses before and after the shaper are fully characterized in amplitude and phase using the frequency-resolved optical gating (FROG) technique developed by Trebino et al. [10]. The method utilises cross correlation between the DUV pulse and a fraction of the well-known fundamental at $800 \mathrm{~nm}$ used to generate the DUV radiation, i.e., an X-FROG pulse-retrieval algorithm. The characterization of spectral amplitudes and phases allows for control over the relative phase of a double pulse in time domain, which is created by a lamellar mirror assembly applied as shaping mask. In our previous research and development work on a prototypical setup, we focused predominately on simulating a theoretical auto-correlation signal of the DUV double pulses [11]. The present contribution goes beyond this by providing the all-optical characterization of amplitude and phase of the tailored waveform, both on the input as well as on the output side. The measured time-frequency spectra are in good agreement with optical simulations.

The structure of the article is in the following order. First, a brief description of the optics used in the $4 f$ shaper setup along with some technical data and diagrams of the apparatus and the femtosecond DUV source used in the experiment is given. It is followed by presenting fully characterized waveforms analysing the effect of modulated spectral amplitudes on the time-frequency distribution of the transmitted femtosecond pulse pair. This contribution is then ended by a discussion in the outlook section shedding some light on future applications of the device and its possible implementation at the free-electron facility FLASH in Hamburg [12]. A coherent soft X-ray laser beam providing intense femtosecond pulses at high repetition rate is at the heart of an upgrade program, which is currently under development at DESY [13-15].

\section{Experiment}

The laser radiation passes the shaper, which consists of a pair of identical gratings $\left(G_{1}, G_{2}\right)$ and identical focusing mirrors $\left(\mathrm{FM}_{1}\right.$ and $\left.\mathrm{FM}_{2}\right)$ used to spatially disperse and re-collimate the spectrum of the ultrashort laser pulse. The central component of the device is an amplitude/phase mask placed in the Fourier plane of the symmetric $4 f$ compressor setup. In the XUV and soft X-ray design of the instrument, there are two additional symmetric focusing mirrors installed in the beam path to decrease the beam footprint on the optics in the center of the shaper setup in the plane perpendicular to the dispersion direction. This reduced footprint in beam direction allows for shorter shaping masks working in grazing incidence, which are easier to manufacture in high quality. The detailed specifications of the installed reflective optics are discussed in Refs. $[11,16]$. In the present DUV experiments, the outer mirror pair reducing the footprint on the mask was replaced by identical flat mirrors because of better overall reflectivity in the deep ultraviolet spectral range. The experimental setup is depicted in Fig. 1.

At the entrance of the $4 f$ line, the input beam coming under a grazing incident angle of $\theta_{i}$ and with a central wavelength $\lambda_{0}$ is initially diffracted at an angle $\theta_{d}$ by the first grating dependent on the grating period $d$. Defining $f$ as the focal length of the following focusing optics, and assuming diffraction-limited focusing, the spatial width of each spectral component in the Fourier plane can be written as

$\Delta x_{0}=2 \ln (2) \frac{\sin \theta_{i} f \lambda_{0}}{\sin \theta_{d} \pi \Delta x_{\text {in }}}$,

where $\Delta x_{\text {in }}$ is defined as the input FWHM beam diameter and $\theta_{i}$ is $6^{\circ}$. The spatial position, $X_{k}$, of each angular frequency component, $\omega_{k}$, can also be defined for a $4 f$ shaper as

$X_{k}=\omega_{k} \alpha$,

with $\alpha$ being a constant defined by the geometry of the $4 f$ line

$\alpha=\frac{\lambda_{0}^{2} f}{2 \pi c d \sin \theta_{d}}$,

where $c$ is the speed of light. For the spectral resolution, one derives

$\frac{\delta \omega}{X_{k}}=\frac{\Delta x_{0}}{\alpha}=\Delta x_{0} \frac{2 \pi c d \sin \theta_{d}}{\lambda_{0}^{2} f}$,

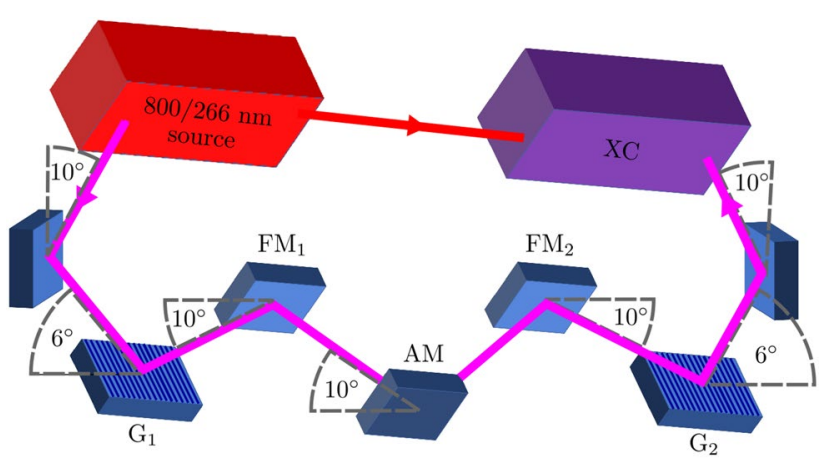

Fig. 1 Simplified diagram of the experimental setup. Key components, i.e., the gratings $G_{1}$ and $G_{2}$, focusing mirrors $\mathrm{FM}_{1}$ and $\mathrm{FM}_{2}$, and the amplitude mask AM are highlighted. The specific grazing angles of incidence in the setup are $6^{\circ}$ for $G_{1}$ and $G_{2}, 10^{\circ}$ for $\mathrm{FM}_{1}, \mathrm{FM}_{2}$ and $\mathrm{AM}$ 
which, through the Fourier transform, corresponds to a shaping window of

$T=4 \ln (2) / \delta \omega=\Delta x_{\text {in }} /|v|$,

where $|v|$ has the dimension of velocity

$v=c d \sin \theta_{i} / \lambda_{0}$.

For a more complete theoretical description of the $4 f$ pulse shaping concept, please see $[4,17,18]$. The performance parameters of the present DUV pulse shaper are summarized in Table 1.

From Eq. (2), it is clear that each spectral component is separated at a different spatial position in the Fourier plane, allowing amplitude and phase transformations to be performed on chosen parts of the dispersed spectrum with a resolution defined by Eq. (4). By placing a mask in

Table 1 Performance parameters of the DUV pulse shaper

Fig. 2 a Simplified sketch of the amplitude mask located in the Fourier plane of the zero-dispersion compressor. Reflective stripes of $100 \mu \mathrm{m}$ width are separated by $150 \mu \mathrm{m}$ gaps. b Surface profile of the amplitude mask recorded by means of white-light interferometry on the area illuminated by the DUV beam. $\mathbf{c}$ Histogram of $\mathbf{b}$. Two typical DUV spectra measured before and after the shaper and smoothed using a spline filter are shown on the top left and top right, respectively

\begin{tabular}{ll}
$T$ & $12.8 \mathrm{ps}$ \\
$v$ & $0.39 \mathrm{~mm} / \mathrm{ps}$ \\
\hline
\end{tabular}

the Fourier plane with a defined complex spectral transformation, $R(\omega)$, and fully defining the input pulse as $E_{\text {in }}$, the output pulse is given by

$E_{\text {out }}(\omega)=R(\omega) E_{\text {in }}(\omega)$,

where $R(\omega)$ contains all information on the spectral and phase transformations. In the present contribution, we used a special reflective lamellar-mirror assembly to periodically shape the spectral amplitudes of the femtosecond DUV pulses, as shown in Fig. 2a.

The focusing mirrors $\mathrm{FM}_{1}$ and $\mathrm{FM}_{2}$ are made from polished silicon, which provides $\sim 90 \%$ reflectivity under grazing incidence for the DUV. The gratings $G_{1}$ and $G_{2}$ are made of BK-7 glass coated with aluminum and blazed at $10.4^{\circ}$. The amplitude mask is made from a polished silicon substrate with reflective stripes of $100 \mu \mathrm{m}$ width and gaps of $150 \mu \mathrm{m}$. The latter were micro-machined using a diamond rotary saw. The resulting surface profile is characterized by means of white-light interferometry on the area illuminated by the DUV beam. The experimental data and the corresponding histogram are shown in Fig. 2b, c, respectively. The analysis gives a root-mean-square (RMS) difference value of $2.1 \mathrm{~nm}$ with respect to a planar surface supporting soft $\mathrm{X}$-ray pulse-shaping applications down to the few-nanometer wavelength scale. One has to keep in mind that the phase errors caused by these spatial imperfection are significantly reduced due to the reflective shaping setup working under grazing incidence. (a)
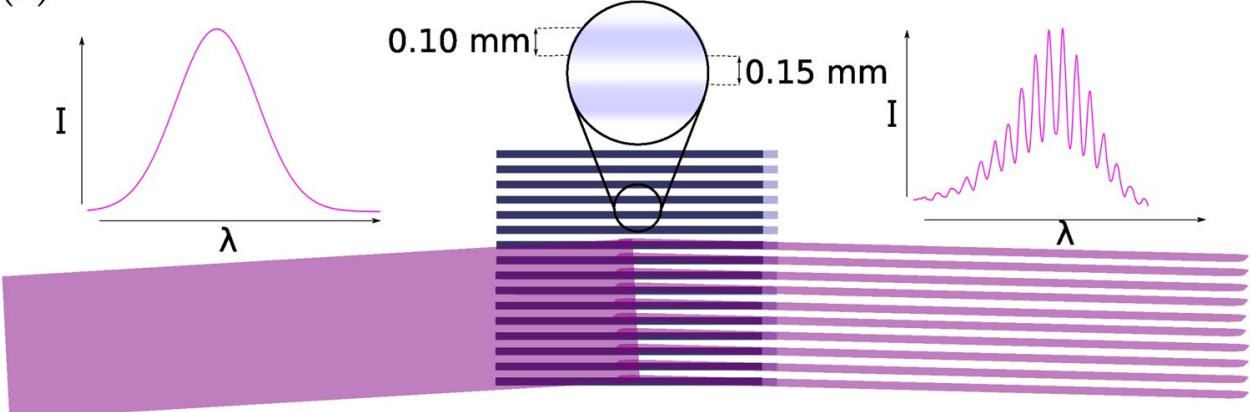

(b)

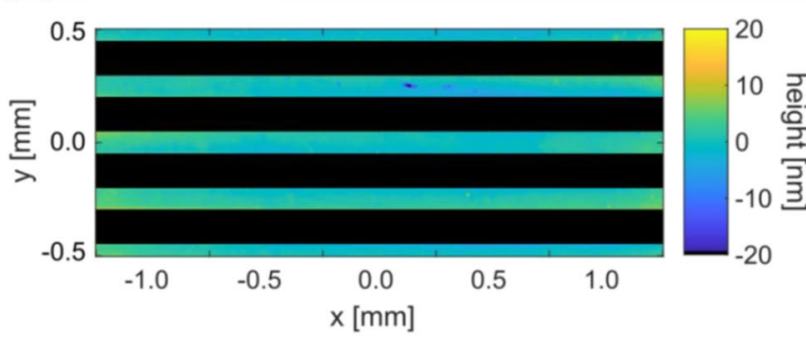

(c)

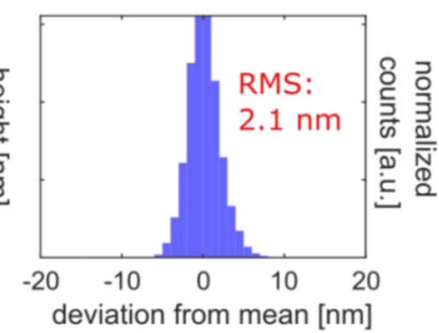


The femtosecond DUV test pulse in this experiment was generated as the third harmonic of an $800 \mathrm{~nm}$ Ti:Sa pulse, using a commercial tripler kit from EKSMA Optics, model FK-800-050-10. Details on third harmonic generation and nonlinear crystals are described elsewhere [18]. The measured FWHM pulse duration and spectral bandwidth of both the fundamental and third harmonic generated are $50 \pm 5 \mathrm{fs}$ $(20 \pm 2.8 \mathrm{~nm})$ and $110 \pm 13 \mathrm{fs}(2.8 \pm 0.2 \mathrm{~nm})$, respectively. The pulse properties of the fundamental were characterized using an 8-50-USB GRENOUILLE from SWAMP Optics with a FROG G-error of 0.1421 and then cross-checked using an autocorrelator and a spectrometer. The properties of the DUV pulses were characterized using the X-FROG technique using the $800 \mathrm{~nm}$ as a well-known reference pulse.
Each pulse was measured ten times to calculate the error as standard deviation. The achieved conversion efficiency from the fundamental to third harmonic was on the order of $4 \%$.

\section{Results and discussion}

In Fig. 3a-f, the retrieved spectrogram, spectra, and phase are displayed for the DUV pulse for the three different experimental scenarios. The retrieval algorithm applied to the recorded X-FROG data is the basic FROG algorithm discussed in detail in Refs. [19, 20]. The unshaped pulse characterized in front of the shaper setup is shown in Fig. 3a, b with an FROG G-error of 0.025 . The femtosecond pulse
Fig. 3 Spectrograms, spectra, and phase for the DUV pulse, retrieved by means of crossfrequency-resolved optical gating (X-FROG) measured before the shaper $(\mathbf{a}, \mathbf{b})$, after the shaper with a plane mirror as phase mask (c, d), and after the shaper using a lamellar-mirror amplitude mask $(\mathbf{e}, \mathbf{f})$. The orange dotted line represents the retrieved spectral phase, the blue dotted line the retrieved spectrum, and the solid red line the measured spectrum. Clearly visible in each figure is a positive linear chirp, that translates from before the shaper to behind the device with both a flat mirror and lamellarmirror assembly installed in the Fourier plane of the shaper. The electric-field envelope is displayed on each spectrogram as a white line. The double pulse in $\mathbf{e}$ is separated in time domain at $630 \pm 24 \mathrm{fs}$

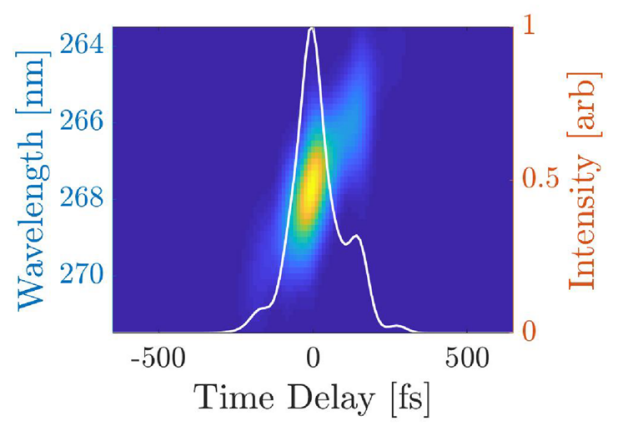

(a) UV before shaper

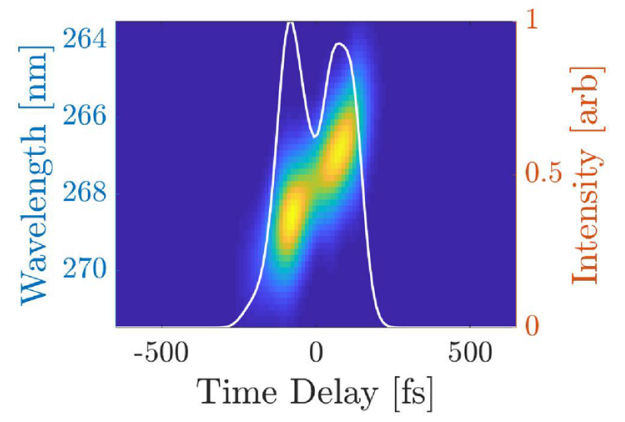

(c) UV after shaper - unshaped

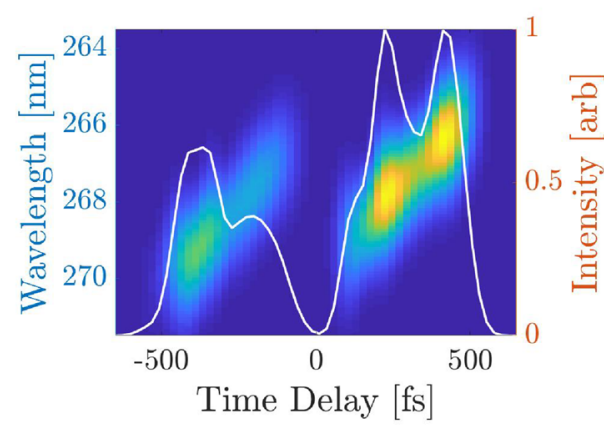

(e) UV after shaper - shaped

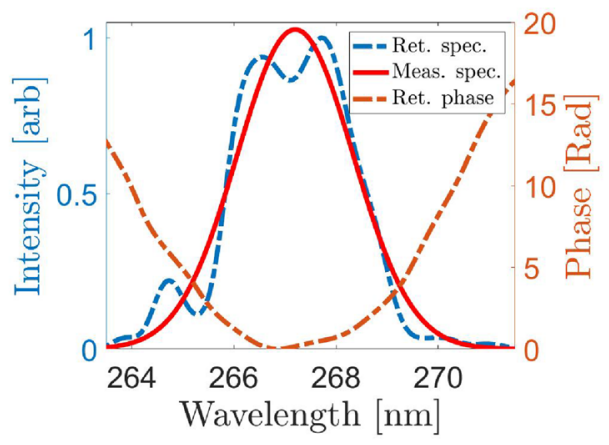

(b) Spectra and phase before shaper

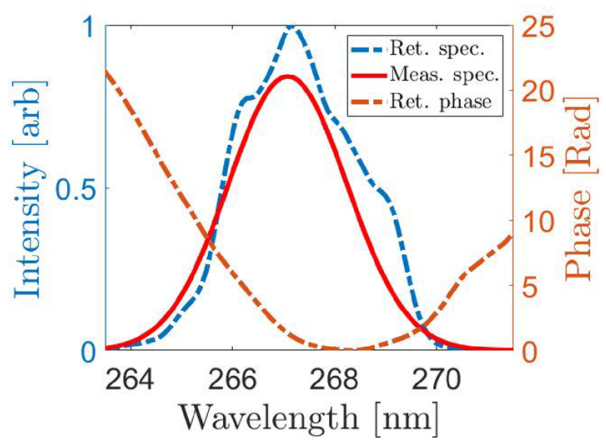

(d) Spectra and phase - unshaped

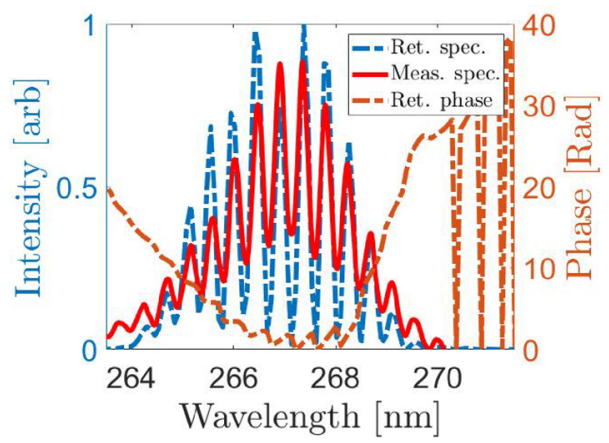

(f) Spectra and phase - shaped 
Fig. 4 Stylized representation of the center-of-mass wavelength oscillations a of the pre- and post-pulse as the amplitude modulation mask moves over the spectra for a $2 \pi$ range (being equivalent to the lamellar-mirror-assembly period of $250 \mu \mathrm{m}$ for lateral movement) in steps of $\pi / 2$. Retrieved center-of-mass wavelength oscillations for the pre- (b) and post-pulse (c) as a function of amplitude-mask position in the Fourier plane

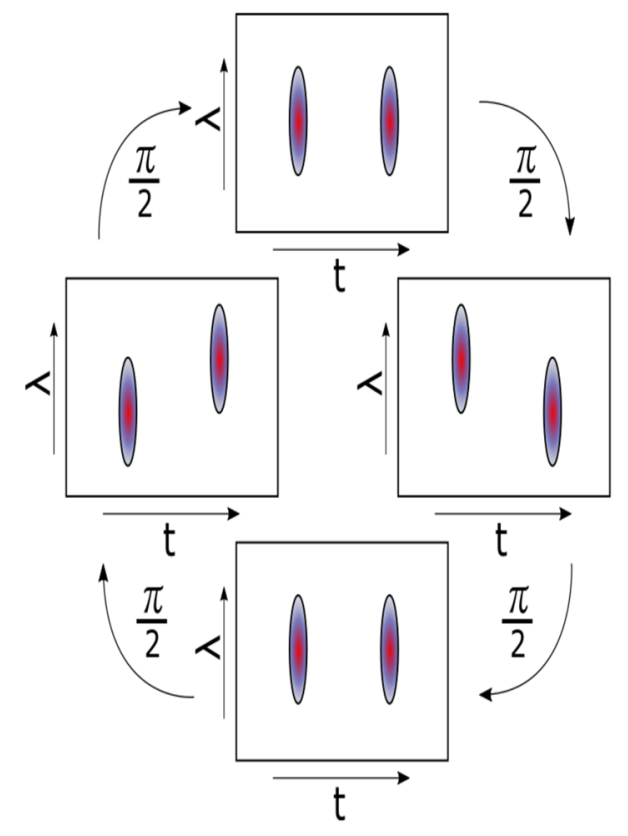

(a) Center-of-mass oscillation diagram

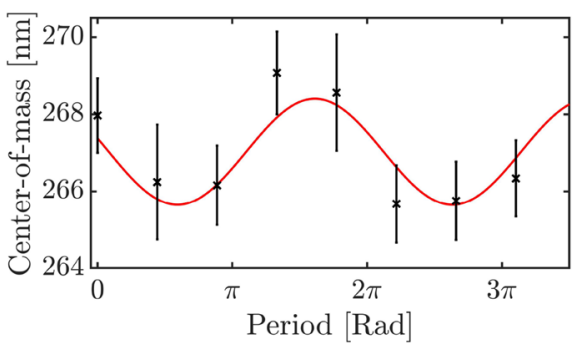

(b) Pre-pulse wavelength oscillations

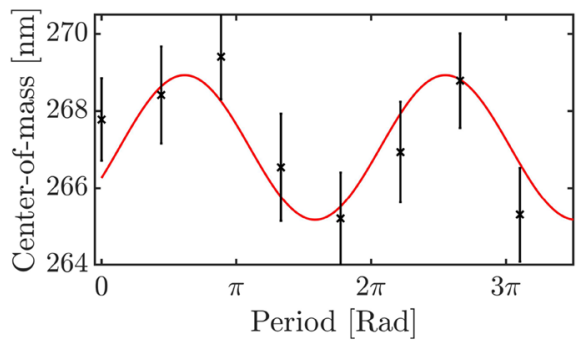

(c) Post-pulse wavelength oscillations after the shaper with no active amplitude modulation performed, i.e., with a plane mirror positioned in the Fourier plane, is shown in Fig. 3c, d with an FROG G-error of 0.027. The effect of the lamellar-mirror amplitude mask performing the shaping transformation is demonstrated in Fig. 3e, f with an FROG G-error of 0.027. The overall agreement between measured and retrieved DUV spectra is reasonable. The differences originate from the low signal-to-noise ratios of the weak DFG signals.

A clear positive linear chirp is observable in all spectrograms (a, c, e), characterized by the retrieved quadratic spectral phases for each pulse (b, d, f). This chirp is inherited from the frequency up-conversion process. It is apparent from the retrieved data describing the unshaped pulses that the shaper introduces a little positive chirp. In Fig. 3c, d, a double temporal feature is present in the pulse, which may stem from an unbalanced grating causing an additional modulation overtone upon the spectral amplitude. This interpretation is supported by the appearance of the same structure also in Fig. 3e, f, showing that this structure is independent of the amplitude mask. We note in passing that each optical component of the complex setup depicted in Fig. 1 is equipped with in-vacuum motorisation for the various optical degrees of freedom, which sums up to a total amount of 37 translations and rotations being optimized as good as possible in the course of the commissioning $[11,16]$.

The targeted amplitude-shaped $266 \mathrm{~nm}$ pulse characterized in Fig. 3e, f shows a clear modulation in the retrieved spectral phase. The modulation period is the same as present in the pulse wavelength spectrum. A closer look at the modulated spectral amplitude of the retrieved shaped pulse, which is the spacing between two neighbouring peaks, gives $0.449 \pm 0.027 \mathrm{~nm}$. This nicely compares to the measured spectral separation of $0.444 \pm 0.003 \mathrm{~nm}$. Taking this modulation into account, the theoretical temporal separation between subsequent pulses should be on the order of $600 \pm 36 \mathrm{fs}$, which is in good agreement with the measured value of $630 \pm 24 \mathrm{fs}$ [11].

After generating a phase-locked DUV double pulse, the next step is to demonstrate coherent-control capabilities of the pulse-shaping device [21]. For an amplitude mask that periodically modulates the pulse spectrum, such control can be achieved by translating the lamellar-mirror assembly along the dispersion plane of the input beam. Shifting the selection of reflected spectra segments will cause the centerof-mass wavelengths of the pre- and post-pulse to oscillate over the period length of the mask, as depicted in Fig. 4a. To that extent, the spectrogram of each sub-pulse was measured in steps of roughly $0.45 \pi$ over a range of $3.6 \pi$, and the retrieved center-of-mass wavelength is plotted in Fig. 4b, c.

As predicted, the center-of-mass wavelength of the preand post-pulse oscillates with a $\pi$ relative-phase difference. The theory behind this prediction can be found in Wollenhaupt et al. [Eq. (6) [22]]. In turn, the relative phases between the pre- and post-pulses can be controlled by means of a specific lamellar-mirror-assembly position [23]. The relative-phase values repeat over a $2 \pi$ period, which corresponds to the spatial mask period of $250 \mu \mathrm{m}$ in the Fourier 
plane of the shaper. It is important to note that the temporal separation between the pre- and post-pulse stays constant in this "open-loop" control protocol [24].

\section{Summary and outlook}

We presented a complete spectral amplitude and phase characterisation of a phase-locked deep UV pulse pair generated by an all-reflective $4 f$ pulse shaper. Using an open-loop coherent-control protocol, the relative phase between the pre- and post-pulse was continuously tuned between 0 and $2 \pi$, while keeping the temporal separation at $\sim 630 \pm 24 \mathrm{fs}$ constant. The operation of this device at significantly shorter wavelengths is mainly determined by the surface quality of the lamellar-mirror assembly. In recent experiments, we could demonstrate that we are indeed able to split soft X-ray beams at $274.7 \mathrm{eV}$ photon energy $\left(\lambda_{0} \approx 4.5 \mathrm{~nm}\right)$ into two replicas with sub-cycle precision and constant relative-phase difference across the beam profile [25]. The demonstration of relative-phase control with grazing-incidence optics is expected to push the wavelength limit for coherent-control applications down into the soft X-ray spectral range providing element specificity in the laser-matter interaction. Research and development towards enabling technology in this field open up new windows of opportunities for coherent soft X-ray pulses emitted from seeded free-electron lasers (FEL) and intense table-top high-harmonic generation (HHG) sources [26-28].

\section{Appendix}

For the FROG retrieval software used in this work, please see: http://www.swampoptics.com/frog-code.html.

Acknowledgements This work was supported by the Deutsche Forschungsgemeinschaft (DFG, German Research Foundation) through the collaborative research center 'Light-induced Dynamics and Control of Correlated Quantum Systems' (SFB-925—project 170620586), the projects KI 482/20-1 and LA $1431 / 5-1$, by the Federal Ministry of Education and Research of Germany (BMBF) through collaborative research projects FSP 302 (05K13GU4) and LoKoFEL (05K2016), and by the Innovation Pool initiative within the Helmholtz Association's research field Matter: 'Enabling Technologies for Compact High Rate Photon Sources' (ECRAPS)

Funding Open Access funding enabled and organized by Projekt DEAL.

Open Access This article is licensed under a Creative Commons Attribution 4.0 International License, which permits use, sharing, adaptation, distribution and reproduction in any medium or format, as long as you give appropriate credit to the original author(s) and the source, provide a link to the Creative Commons licence, and indicate if changes were made. The images or other third party material in this article are included in the article's Creative Commons licence, unless indicated otherwise in a credit line to the material. If material is not included in the article's Creative Commons licence and your intended use is not permitted by statutory regulation or exceeds the permitted use, you will need to obtain permission directly from the copyright holder. To view a copy of this licence, visit http://creativecommons.org/licenses/by/4.0/.

\section{References}

1. C.W. Hillegas, J.X. Tull, D. Goswami, D. Strickland, W.S. Warren, Femtosecond laser pulse shaping by use of microsecond radio-frequency pulses. Opt. Lett. 19, 737-739 (1994)

2. A.M. Weiner, Femtosecond pulse shaping using spatial light modulators. Rev. Sci. Instrum. 71, 1929-1960 (2000)

3. M.A. Jakob, M. Namboodiri, M.J. Prandolini, T. Laarmann, Generation and characterization of tailored MIR waveforms for steering molecular dynamics. Opt. Express 27, 26979-26988 (2019)

4. A. Monmayrant, S. Weber, B. Chatel, A newcomer's guide to ultrashort pulse shaping and characterization. J. Phys. B At. Mol. Opt. Phys. 43, 103001 (2010)

5. B.J. Pearson, T.C. Weinacht, Shaped ultrafast laser pulses in the deep ultraviolet. Opt. Express 15, 4385-4388 (2007)

6. M. Hacker, G. Stobrawa, R. Sauerbrey, T. Buckup, M. Motzkus, M. Wildenhain, A. Gehner, Micromirror SLM for femtosecond pulse shaping in the ultraviolet. Appl. Phys. B 76, 711-714 (2003)

7. A. Rondi, J. Extermann, L. Bonacina, S. Weber, J.-P. Wolf, Characterization of a mems-based pulse-shaping device in the deep ultraviolet. Appl. Phys. B 96, 757-761 (2009)

8. D. Kiselev, P.M. Kraus, L. Bonacina, H.J. Wörner, J.-P. Wolf, Direct amplitude shaping of high harmonics in the extreme ultraviolet. Opt. Express 20, 25843-25849 (2012)

9. S. Usenko, A. Przystawik, L.L. Lazzarino, M.A. Jakob, F. Jacobs, C. Becker, C. Haunhorst, D. Kip, T. Laarmann, Split-and-delay unit for FEL interferometry in the XUV spectral range. Appl. Sci. 7, 544 (2017)

10. R. Trebino, Frequency-Resolved Optical Gating: the Measurement of Ultrashort Laser Pulses (Springer Science \& Business Media, Berlin, 2012)

11. L.L. Lazzarino, M.M. Kazemi, C. Haunhorst, C. Becker, S. Hartwell, M.A. Jakob, A. Przystawik, S. Usenko, D. Kip, I. Hartl, T. Laarmann, Shaping femtosecond laser pulses at short wavelength with grazing-incidence optics. Opt. Express 27, 13479-13491 (2019)

12. B. Faatz, E. Plönjes, S. Ackermann, A. Agababyan, V. Asgekar, V. Ayvazyan, S. Baark, N. Baboi, V. Balandin, N. Von Bargen et al., Simultaneous operation of two soft X-ray free-electron lasers driven by one linear accelerator. New J. Phys. 18, 062002 (2016)

13. S. Ackermann, A. Azima, S. Bajt, J. Bödewadt, F. Curbis, H. Dachraoui, H. Delsim-Hashemi, M. Drescher, S. Düsterer, B. Faatz et al., Generation of coherent 19- and 38-nm radiation at a free-electron laser directly seeded at $38 \mathrm{~nm}$. Phys. Rev. Lett. 111, 114801 (2013)

14. K. Hacker, R. Molo, S. Khan, L. Lazzarino, C. Lechner, T. Maltezopoulos, T. Plath, J. Rossbach, S. Ackermann, J. Bödewadt et al., Measurements and simulations of seeded electron microbunches with collective effects. Phys. Rev. Spec. Top. Accel. Beams 18, 090704 (2015)

15. T. Plath, P. Amstutz, J. Bödewadt, G. Brenner, N. Ekanayake, B. Faatz, K. Hacker, K. Honkavaara, L.L. Lazzarino, C. Lechner et al., Free-electron laser multiplex driven by a superconducting linear accelerator. J. Synchrotron Radiat. 23, 1070-1075 (2016) 
16. L.L. Lazzarino, Design and commissioning of an xuv and soft -Xray fel pulse shaper. Ph.D. thesis (Staats-und Universitätsbibliothek Hamburg Carl von Ossietzky 2018)

17. C. Froehly, B. Colombeau, M. Vampouille, Shaping and analysis of picosecond light pulses. Progr. Opt. 20, 63-153 (1983)

18. R.W. Boyd, Nonlinear Optics (Academic Press, New York, 2020)

19. K.W. DeLong, R. Trebino, Improved ultrashort pulse-retrieval algorithm for frequency-resolved optical gating. J. Opt. Soc. Am. A 11, 2429-2437 (1994)

20. K.W. DeLong, D.N. Fittingho, R. Trebino, B. Kohler, K. Wilson, Pulse retrieval in frequency-resolved optical gating based on the method of generalized projections. Opt. Lett. 19, 2152-2154 (1994)

21. A. Wituschek, L. Bruder, E. Allaria, U. Bangert, M. Binz, R. Borghes, C. Callegari, G. Cerullo, P. Cinquegrana, L. Giannessi et al., Tracking attosecond electronic coherences using phasemanipulated extreme ultraviolet pulses. Nat. Commun. 11, 1-7 (2020)

22. M. Wollenhaupt, A. Präkelt, C. Sarpe-Tudoran, D. Liese, T. Bayer, T. Baumert, Femtosecond strong-field quantum control with sinusoidally phase-modulated pulses. Phys. Rev. A 73, 063409 (2006)

23. T. Laarmann, I. Shchatsinin, P. Singh, N. Zhavoronkov, C.P. Schulz, I.V. Hertel, Femtosecond pulse shaping as analytic tool in mass spectrometry of complex polyatomic systems. J. Phys. B At. Mol. Opt. Phys. 41, 074005 (2008)

24. T. Laarmann, I. Shchatsinin, P. Singh, N. Zhavoronkov, M. Gerhards, C. Schulz, I. Hertel, Coherent control of bond breaking in amino acid complexes with tailored femtosecond pulses. J. Chem. Phys. 127, 201101 (2007)

25. S. Usenko, A. Przystawik, M.A. Jakob, L.L. Lazzarino, G. Brenner, S. Toleikis, C. Haunhorst, D. Kip, T. Laarmann, Attosecond interferometry with selfamplified spontaneous emission of a freeelectron laser. Nat. Commun. 8, 1-6 (2017)

26. T. Laarmann, H.N. Chapman, S. Bajt, Moving the frontier of quantum control into the soft X-ray spectrum. Int. J. Opt. 417075, $1-4(2011)$

27. A. Wituschek, L. Bruder, E. Allaria, U. Bangert, M. Binz, C. Callegari, P. Cinquegrana, M. Danailov, A. Demidovich, M. Di Fraia et al., High-gain harmonic generation with temporally overlapping seed pulses and application to ultrafast spectroscopy. Opt. Express 28, 29976-29990 (2020)

28. J. Rothhardt, S. Hädrich, Y. Shamir, M. Tschnernajew, R. Klas, A. Hoffmann, G.K. Tadesse, A. Klenke, T. Gottschall, T. Eidam et al., High-repetition-rate and high-photon flux $70 \mathrm{eV}$ high-harmonic source for coincidence ion imaging of gas-phase molecules. Opt. Express 24, 18133-18147 (2016)

Publisher's Note Springer Nature remains neutral with regard to jurisdictional claims in published maps and institutional affiliations. 